\title{
Resectable Extrahepatic Bile Duct Adenocarcinoma
}

National Cancer Institute

\section{Source}

National Cancer Institute. Resectable Extrahepatic Bile Duct Adenocarcinoma. NCI

Thesaurus. Code C156911.

Extrahepatic bile duct adenocarcinoma that is amenable to surgical resection. 